\title{
Association between schizophrenia and DRD3 or HTR2 receptor gene variants
}

\author{
S Baritaki ${ }^{1}$, E Rizos $^{2}$, A Zafiropoulos ${ }^{1}$, G Soufla ${ }^{1}$, K Katsafouros ${ }^{2}$, V Gourvas ${ }^{1}$ \\ and DA Spandidos*,1 \\ ${ }^{1}$ Department of Virology, Medical School, University of Crete, Heraklion, Crete, Greece; ${ }^{2}$ Athens Dromokaition \\ Psychiatric Hospital, Greece
}

Schizophrenia is a severe and common psychiatric disorder afflicting $1 \%$ of the world population. A role of many neurotransmitter receptors in schizophrenia was suggested by an association with several polymorphisms located in their coding regions. In this study we examined the contribution of the T-102C and A-206C transitions in the 5-HTR2a and DRD3 receptor genes respectively to genetic susceptibility and phenotypic expression of schizophrenia disorder within the Greek population. We determined by PCR and RFLP analysis the genotype for the above polymorphisms in 114 schizophrenic hospitalized individuals and 192 control samples. In contrast to previous reports from large European multicentre studies, which indicate significant correlation between schizophrenia and C-102 allele of the T-102C polymorphism, in this study we observed a statistically significant overall association between the disorder and allele T-102 $(P<0.0001$, odds ratio $(O R)=2.11,95 \% \mathrm{Cl}=1.48-3.02)$. We also found a highly significant excess of the $\mathrm{T}-102 / \mathrm{C}-102$ and $\mathrm{C}-102 / \mathrm{C}-102$ genotypes in the normal group $(P<0.001)$. Comparison of the patients with the controls for the DRD3 polymorphism (A-206G transition) showed marginally nonsignificant differences in the genotypic $(P=0.054)$ and no significance in the allelic $(P=0.163)$ frequencies. However, the $A-206 /$ A-206 genotype seems to positively contribute to the disorder appearance, when compared to A-206/ $\mathrm{G}-206$ as genotype base line risk $(P=0.016, \mathrm{OR}=1.88,95 \% \mathrm{Cl}=1.09-3.26)$. In conclusion, from genetic association analysis of this schizophrenic population, a significant association is clearly determined between the HTR2 genetic polymorphism and the presence of schizophrenic disorder, manifested as increased risk of schizophrenia for carriers of the T-102 allele.

European Journal of Human Genetics (2004) 12, 535-541. doi:10.1038/sj.ejhg.5201180

Published online 14 April 2004

Keywords: schizophrenia; serotonin receptor; dopamine receptor; polymorphism; association study; RFLP

Introduction

Schizophrenia is a clinical syndrome of the brain that manifests with multiple signs and symptoms thought perception, emotion, movement and behavior. These manifestations show considerable diversity among patients

${ }^{*}$ Correspondence: Professor DA Spandidos, Laboratory of Virology, Medical School, University of Crete, PO Box 1527, Heraklion 711 10, Crete, Greece. Tel: + 302810 394631; Fax: +30 2810 394759; E-mail: spandidos@spandidos.gr Received 24 July 2003; revised 12 November 2003; accepted 21 January 2004 and the final prognosis of the disorder is severe and usually long lasting. Estimates of the incidence and prevalence of schizophrenia depend on the criteria for diagnosis and the population surveyed. The incidence also varies with age, the highest rates occurring in young men and 35-39-yearold women. The lifetime risk of developing schizophrenia is between 7 and 9 per 1000 of the population.

Several types of evidence suggest that genes involved in serotoninergic or dopaminergic neurotransmission are possible factors in the pathogenesis of schizophrenia, extensively studied because of the role of their protein products as sites for antipsychotic drug action. 
Psychopharmacology and biochemical research introduced dopamine hypothesis as a major factor in the development of the disease. ${ }^{1}$ It has been postulated that increases in dopamine neurotransmission after transmethylation of dopamine underlie schizophrenia. Dopaminergic agonists worsen schizophrenic symptoms in patients and produce schizophrenia-like psychosis in normal individuals (amphetamine hypothesis). On the basis of main pharmacological profiles, Seeman and Lee $^{2}$ demonstrated that the potency of neuroleptics directly correlates with their affinity to dopamine receptors, especially to type 2 (DRD2) or type 3 (DRD3) receptors, thus promoting these proteins and their corresponding genes as candidates for schizophrenia.

One of the better-studied actions of the serotoninergic systems is the regulation of the dopaminergic neurotransmission, which influence a number of emotional functions and behaviors. Their involvement in the pathogenesis of the schizophrenic disorder was introduced by the observation that the 5-hydroxytryptamine receptors (5-HTR1 to 4) act as sites for antipsychotic drug action as they can be blocked by the lysergic acid diethylamide (LSD) or clozapine. $^{3}$

The silent mutation T-102C (Ser34Ser) within the coding region of the 5-hydroxytryptamine type $2 \mathrm{a}$ (5-HTR2a) receptor gene as well as the A-206G transition in the sequence of the dopamine type 3 (DRD3) receptor gene that leads to a Ser9Gly amino-acid substitution in the Nterminal extracellular domain of the receptor are genetic polymorphisms previously implicated to confer susceptibility to psychiatric disorders. ${ }^{4,5}$ European multicentre studies indicate significant association between schizophrenia and C-102 variant of the T-102C polymorphism ${ }^{6}$ as well as an increased homozygosity of either allele of the DRD3 polymorphism. ${ }^{7}$ However, negative associations have widely been reported, especially for the DRD3 Ser9Gly transition. $^{8-10}$

As a continuation of these studies, we further genotyped the above 5-HTR2a and DRD3 receptor gene polymorphisms in Greek samples, in order to investigate the distribution of the allelic variants within the population and to examine their putative correlation with schizophrenia.

\section{Materials and methods Human subjects}

Unrelated individuals ( $n=114)$ (67 males and 47 females, mean age $50.6 \pm 13.39$ years), who fulfilled the DSM-IV criteria for schizophrenia were recruited among hospitalized patients of the Athens Dromokaition Psychiatric Hospital, Greece. The diagnosis of schizophrenia was also based on all available clinical information (eg structured interview). Blood samples were collected between February 2003 and June 2003. Healthy individuals $(n=192)(118$ males and 74 females, mean age $45.1 \pm 8.7$ years) recruited at random among prospective blood donors, served as controls. All the normal subjects were obtained from blood transfusion service that excludes individuals on medication, but does not screen for a family history of psychiatric morbidity or other known genetic predisposition.

The genotype distribution among the patient group was also correlated with clinical characteristics, including sex, family history of the disease, clinical type of schizophrenic disorder and the age of disease diagnosis. The last parameter refers to the first onset of schizophrenic symptoms and ranges between 14 and 60 years (mean age $25.5 \pm 9.2$ ). For statistical purposes, the majority of patients with paranoid schizophrenia were classified as 'paranoid type' and all the others (disorganized, catatonic and residual) were grouped as 'other type'.

All patients and controls were unrelated Greek residents. Ethnic bias within the population studied was minimized by excluding subjects from outside Greece. Informed consent was obtained from all the individuals that participated in the study.

\section{Polymerase chain reaction}

Genomic DNA was extracted from $10 \mathrm{ml}$ EDTA treated venous blood samples using the standard phenol/chloroform protocol. DNA purity was assessed by a UV/VIS spectrophotometer estimating the $\mathrm{A}_{260} / \mathrm{A}_{280}$ ratio.

Genotyping for the Ser9Gly (A-206G) or T-102C polymorphisms in the dopamine DRD3 or serotonin HTR2 receptor genes respectively was originally performed by polymerase chain reaction using pairs of external primers framing the regions surrounding the polymorphism's genetic sites. The primers F 5'-GCTCTATCTCCAACTCTCACA-3' and R 5'-AAGTCTACTCACCTCCAGGTA-3' were used to amplify a $463 \mathrm{bp}$ fragment derived from exon 1 of the DRD3 receptor gene carrying the Á/G base transition at the 206-amplimere position. ${ }^{7}$ A genomic DNA fragment of $342 \mathrm{bp}$ corresponding to nucleotides -24 to 318 of the serotonin HTR2 receptor gene and carrying the T102C polymorphic site were amplified using a modification of the published set of primers: ${ }^{6}$ F $5^{\prime}$-TCTGCTACAAGTTCTGGCTT-3', R 5'-CTGCAGCTTTTTCTCTAGGG-3'. The primers were designed from the complete coding sequences of the appropriate genes using the Primer Express 1.0 software. The sequences chosen from the list generated by Primer Express were purchased from MWGBiotech.

For both polymorphisms genomic DNA (50 ng) was amplified in total volume of $20 \mu \mathrm{l}$ of the following PCR reaction mixture: $50 \mathrm{mM} \mathrm{KCl,} 20 \mathrm{mM}$ Tris- $\mathrm{HCl}(\mathrm{pH} 8.4$ ), $1.5 \mathrm{mM} \mathrm{MgCl}_{2}, 0.2 \mathrm{mM}$ of each deoxynucleotide triphosphate (dNTPs), $0.25 \mu \mathrm{M}$ of each oligonoucleotide primer and $0.6 \mathrm{U}$ of recombinant Taq DNA polymerase (Invitrogen). After initial denaturation at $94^{\circ} \mathrm{C}$ for $3 \mathrm{~min}$, the PCR samples were subjected for 35 cycles, each cycle consisted 
of $40 \mathrm{~s}$ denaturation at $94^{\circ} \mathrm{C}, 45 \mathrm{~s}$ annealing at $57^{\circ} \mathrm{C}$ and $45 \mathrm{~s}$ extension at $72^{\circ} \mathrm{C}$. The reaction was completed with 10 -min final extension of PCR products at $72^{\circ} \mathrm{C}$. Appropriate negative and positive controls were included.

Each reaction mixture $(8 \mu \mathrm{l})$ was electrophorezed on a $2 \%$ agaroze gel and analyzed for confirmation of the presence of the correct DNA amplimere.

\section{RFLP analysis}

The allele assessment for both polymorphisms was performed using specific RFLP analysis. ${ }^{11,7}$ PCR products $(8 \mu \mathrm{l})$ corresponding to 342 or $463 \mathrm{bp}$ DNA fragments were cleaved by $5 \mathrm{U}$ of $\mathrm{MspI}$ or $1.5 \mathrm{U}$ of $\mathrm{MscI}$ restriction endonucleases (New England Biolabs, USA) respectively, at $37^{\circ} \mathrm{C}$ for $4 \mathrm{~h}$. MscI enzyme recognizes and cleaves the TGG $\downarrow$ CCA sequence in blunt ends, whereas MspI endonuclease cleaves the $\mathrm{C} \downarrow$ CGG sequence leaving $5^{\prime}$ protruding ends. Each reaction was performed in total volume of $15 \mu \mathrm{l}$ using the appropriate reaction buffer.

Digestion of the $342 \mathrm{bp}$ PCR amplimere with MspI yields a 342 bp product for the wild-type allele (T-102), and 126 and $216 \mathrm{bp}$ products for the mutant allele (C-102). Similarly, digestion of the $463 \mathrm{bp}$ PCR product with MscI yields 305, 111 and $47 \mathrm{bp}$ products for the wild-type allele (A-206), and 206, 111, 99, $47 \mathrm{bp}$ products for the mutant allele (G-206).

For each polymorphism the different patterns of the digested DNA fragments were analyzed by separation on a 2.5\% EtBr stained agarose gel.

\section{Statistical analysis}

Genotype distributions for each polymorphism were first compared to predictable values from Hardy-Weinberg equilibrium. Statistical analysis of allele and genotype frequencies for normal and schizophrenic groups was performed using $\chi^{2}$ statistics (Pearson test). The same test was also used for the genotype correlation with clinical characteristics including sex, family history and type of schizophrenic disorder. As the age score of symptoms onset was not normally distributed, the comparison of this clinical characteristic for the three genotype groups of each polymorphism was evaluated using nonparametric Kruskal-Wallis tests. Furthermore, the allele presence (homozygous or heterozygous)/absence and the homozygosity (for either allele)/heterozygosity was statistically evaluated for each polymorphism (Pearson analysis).

In all cases, $P$-values less than 0.05 were considered to be statistically significant. Odds ratios (ORs) reported in the Result section were derived from Pearson $\chi^{2}$ analysis. All analyses were performed using SPSSv10 (SPSS Inc., Chicago, IL, USA).

\section{Results}

HTR2 receptor gene polymorphism in control and schizophrenic subjects

The allele frequencies and genotypes corresponding to the T-102C transition in the HTR2 receptor gene that is located on the long arm of chromosome 13 were analyzed in 112 schizophrenic hospitalized individuals and 192 control samples by restriction endonuclease digestion. In all samples currying the T-102C transition, an MspI restriction site was found within the corresponding $342 \mathrm{bp}$ PCR product, resulting in a 216 and a $126 \mathrm{bp}$ cleaved DNA fragments (Figure 1).

The genotypic and allele frequencies for HTR2 (T-102C) polymorphism are shown in Table 1 . Genotype frequency distributions were in agreement with the Hardy-Weinberg equilibrium in all sample groups. Comparison of the genotype frequencies between schizophrenic and normal samples showed a higher percentage of C-102/C-102 homozygotes (25\%) as well as T-102/C-102 heterozygotes $(51.1 \%)$ in the control group $(P<0.001)$. We also established statistically remarkable difference in the allelic frequencies indicating a closed overall association between the disorder and allele T-102 $(P<0.0001, \mathrm{OR}=2.11,95 \%$ $\mathrm{CI}=1.48-3.02)$. We further investigated whether or not $\mathrm{T}-$ 102/T-102 and T-102/C-102 genotypes have different OR values by using C-102/C-102 as genotype baseline risk. Our findings showed that T-102 homozygotes were significantly pronounced within patients $(P=0.0001$, OR $=3.59$, $95 \% \mathrm{CI}=1.71-7.59)$, whereas heterozygotes did not present a considerable difference.

Statistical analysis of the correlation between parameters such as sex, age of the first onset of schizophrenic symptoms, subtype of the schizophrenic disorder (paranoid, disorganized, catatonic and residual) and presence or absence of heredity history within the family members with certain HTR2 genotype, showed no significant results in the group of patients (Table 2). However, heterozygotes compared to homozygotes (T-102/T-102, A-102/A-102)

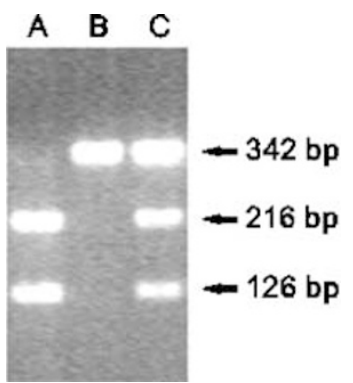

Figure 1 Cleavage of $342 \mathrm{bp}$ PCR product framing the T102C polymorphic site in the serotonin HTR2 receptor gene, by the $\mathrm{Mspl}$ restriction endonuclease. Lane A: DNA band pattern of a C-102/C-102 mutant-type homozygote. Lane B: DNA band pattern of a T-102/T-102 wild-type homozygote. Lane C: DNA band pattern of a T-102/C-102 heterozygote. 
Table 1 Allele and genotypic frequencies in the control and the schizophrenic patient groups

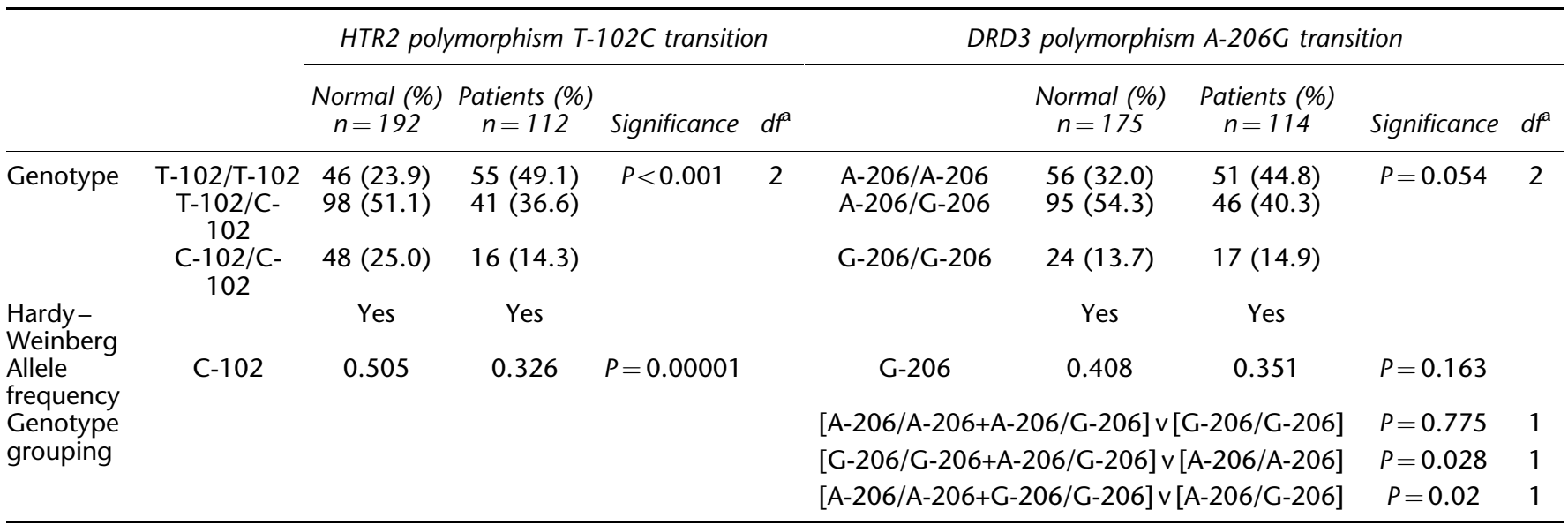

${ }^{\mathrm{a}}$ Degrees of freedom.

Table 2 Incidence of HTR2 and DRD3 polymorphisms in association with clinical parameters within the schizophrenic patient group

\begin{tabular}{|c|c|c|c|c|c|c|c|c|c|}
\hline & \multirow{2}{*}{ Genotype } & \multicolumn{4}{|c|}{ HTR2 polymorphism T-102C transition } & \multicolumn{4}{|c|}{ DRD3 polymorphism A-206G transition } \\
\hline & & $T-102 / T-102$ & $T-102 / C-102$ & C-102/C-102 & $p^{a}$-value & $A-206 / A-206$ & $A-206 / G-206$ & G-206/G-206 & $P^{\mathrm{a}}$-value \\
\hline \multirow[t]{2}{*}{ Sex } & Male & 30 & 28 & 8 & $0.294, \mathrm{df}=2$ & 32 & 26 & 9 & $0.716, \mathrm{df}=2$ \\
\hline & Female & 25 & 13 & 8 & & 19 & 20 & 8 & \\
\hline \multirow{2}{*}{ Type } & Paranoid & 37 & 29 & 13 & $0.558, \mathrm{df}=2$ & 33 & 34 & 13 & $0.507, \mathrm{df}=2$ \\
\hline & Other type & 18 & 12 & 3 & & 18 & 12 & 4 & \\
\hline \multirow{3}{*}{ History } & Heredity & 16 & 17 & 2 & $0.094, \mathrm{df}=2$ & 14 & 18 & 4 & $0.345, \mathrm{df}=2$ \\
\hline & No heredity & 39 & 24 & 14 & & 37 & 28 & 13 & \\
\hline & Age of diagnosis ${ }^{b}$ & $26.9 \pm 1.4$ & $22.7 \pm 0.9$ & $27.1 \pm 2.2$ & $0.083^{c}$ & $25.0 \pm 1.2$ & $25.5 \pm 1.4$ & $24.6 \pm 1.4$ & $0.938^{c}$ \\
\hline
\end{tabular}

apearson $\chi^{2}$ test.

${ }^{\mathrm{b}}$ Mean age of diagnosis \pm SEM (standard error of mean).

${ }^{\mathrm{c}}$ Kruscal-Wallis.

show earlier onset of first psychotic symptoms independently of the type of the schizophrenic disorder (test Kruscal-Wallis $P=0.027$ or 0.015 ANOVA test). This observation is also confirmed by the lower mean age of first schizophrenic symptoms in heterozygotes $(22.7 \pm 0.9)$ compared to that evaluated in homozygotes $(\sim 27)$ (Table 2).

\section{DRD3 receptor gene polymorphism in control and schizophrenic subjects}

The second polymorphism (A-206G) that we observed within the exon 1 of DRD3 receptor gene was analyzed by MscI restriction endonuclease digestion of $463 \mathrm{bp}$ PCR fragment, since an additional MscI restriction site was introduced by the A-206G transition. The MscI cleavage pattern was used for genotyping of the DRD3 polymorphism in 114 schizophrenic patients and 175 controls (Figure 2).

The genotypic and allele frequencies for this DRD3 polymorphism are shown in Table 1. Genotype frequency distributions were in agreement with the Hardy-Weinberg equilibrium in all sample groups. Comparison of the patient subjects with the controls for the A-206G transition showed marginal differences in the genotypic $(P=0.054)$ but not in the allelic $(P=0.163)$ frequencies. For further investigation, we performed independent Pearson $\chi^{2}$ tests in order to access all possible genotype groupings using sequentially the A-206/A-206, A-206/G-206 and G-206/G206 genotypes as baseline risk. The combination of the A206/G-206 and G-206/G-206 genotypes versus A-206/A-206 


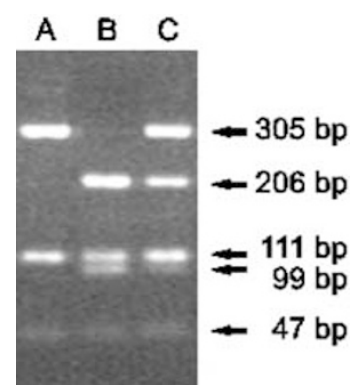

Figure $2 \mathrm{Mscl}$ enzymatic digestion of $463 \mathrm{bp}$ fragment derived from exon 1 of DRD3 receptor gene carrying the Á/ $\mathrm{G}$ base transition. Lane (a) DNA band pattern of an A-206/ A-206 wild-type homozygote. Lane (b) DNA band pattern of a G-206/G-206 mutant-type homozygote. Lane (c) DNA band pattern of an A-206/G-206 heterozygote.

revealed statistically considerable differences $(P=0.028$, $\mathrm{OR}=0.58, \quad>95 \% \mathrm{CI}=0.35-0.97)$, although the best statistical significance was obtained using as baseline risk the factor A-206/G-206 $(P=0.02, \quad$ OR $=1.76, \quad>95 \%$ $\mathrm{CI}=1.06-2.91$ ), which suggests higher susceptibility of both homozygous genotypes for schizophrenic disorder. No other genotype grouping gave statistically significant results. Furthermore, analysis on individual OR values of G206/G-206 and A-206/A-206 genotypes using A-206/G-206 as the genotype baseline risk, provided significant differences for the wild-type homozygous genotype $(P=0.016$, $\mathrm{OR}=1.88,95 \% \mathrm{CI}=1.09-3.26$ ), whereas no variation was observed for the relevant mutant genotype.

Regarding the incidence and the association of the DRD3 polymorphism with the sex of the patients, the subtype of the schizophrenic disorder, the family history and the age of the first onset of the schizophrenic symptoms, no statistically significant correlation was also established (Table 2).

\section{Discussion}

The etiology of schizophrenia remains unknown. Genetic factors are important possibly in all cases, but it is not clear which genes are deviant and how they contribute to pathophysiology of the disease. The search for a genetic risk factor has been examined through studies of twins of families and the children of schizophrenic parents who are adopted by others. Adoption studies showed strong evidence of genetic causes in the etiology of the disease. However, the mode of inheritance appears complex and non-Mendelian. Various genetic models of schizophrenia have proposed the action of a number of genes, which may co-act or interact with each other. ${ }^{12,13}$

Candidate genes for schizophrenia (genes that encode products with neurobiological function such as neurotransmitter receptors or enzymes) are commonly studied by way of association studies. Pharmacological profiles have divided dopamine receptors into DRD1, DRD2, DRD3, DRD4 and DRD5 subtypes. DRD2 has been extensively studied as it shows high affinity for neuroleptics binding. ${ }^{2}$ DRD3 is similar to DRD2 in that it shares common intronic sequences, cellular signaling pathways and has a similar affinity for typical neuroleptics. ${ }^{14,15}$ However, unlike DRD2, it shows a higher affinity for atypical neuroleptics. ${ }^{16}$ DRD3 has a more restricted area of expression in the brain, which is known to be associated with cognitive and emotional function. ${ }^{17}$

Association studies between DRD2 $2^{18,19}$ and $\mathrm{DRD}^{20}$ receptor gene polymorphisms and schizophrenic susceptibility have been carried out with conflicting results. The positive contribution of the A-206G transition within exon 1 of the DRD3 receptor gene was first proposed by Lannnfelt ${ }^{21}$ and Crocq. ${ }^{20}$ More recent European multicenter reports also indicate the existence of a significant correlation between increased homozygosity of either DRD3 allele and the disease; however, they present no evidence of an allelic association. ${ }^{7,22,23}$ Although, controversial conclusions have been reported by a significant number of other groups, which failed to replicate these findings of association with homozygosity of the Ser9Gly variant. ${ }^{24,25,8,9}$ In addition, thorough studies on the characterization, mutation detection and association analysis of alternative promoters and 5'UTRs of the human DRD3 gene in schizophrenia imply that neither the coding nor the regulatory region of DRD3 plays a major role in predisposition to schizophrenia. ${ }^{10}$ On the contrary, the contribution of the A-206G transition to the development of Tardive Dyskinesia (TD) in schizophrenic patients treated with antipsychotics has been maintained unanimously. ${ }^{26,27}$

In the present study we observed a marginally nonsignificant excess in the percentages of both homozygote genotypes in the patient group $(P=0.054)$, although the estimation of the individual OR values of G-206/G-206 and A-206/A-206 genotypes using A-206/G-206 as a genotype baseline risk, revealed statistically significant association for the wild-type homozygotes, $(P=0.016, \mathrm{OR}=1.88,95 \%$ $\mathrm{CI}=1.09-3.26)$, indicating indirectly a higher risk of this genotype to develop schizophrenic disorder. These results are in partial agreement with previously reported data; ${ }^{7,20}$ however, comparison of the allele frequencies between the two groups did not show statistically significant differences $(P=0.163)$, possibly because both alleles are common in the population and therefore only certain genotypic combinations might lead to susceptibility for schizophrenia. The last statement might be generalized, as no considerable geographical differences, concerning the evaluated frequencies for both DRD3 alleles, were obtained between Greece and other European countries, either in control or patient groups. ${ }^{7}$

Additionally, in contrast to previously published studies indicating a significant excess of homozygosity of either 
allele in patients with a positive family history of the disease and in males, ${ }^{28,29}$ as well as a positive correlation of G-206 allele with earlier age of symptoms onset, particularly in males ${ }^{22,30}$, our data do not support any of these associations.

Serotonergic deficiency in schizophrenia was proposed in the early 1950s. LSD, which is chemically similar to serotonin and occupies serotonin receptor sites, was proposed as a model for psychosis. Some of the most intriguing data suggesting that serotonin may play a role in schizophrenia come from studies that focused on the mechanism of action of atypical antipsychotics, such as clozapine. Clozapine excess of weak D2 affinity also blocks 5-HTR1, 5-HTR2 and 5-HTR3 receptors and like many antidepressants it also produces a subsequent downregulation of 5-HT2 receptors. This finding led to speculation that clozapine's unique antipsychotic effects could also be due to 5-HTR2 blockade. ${ }^{31}$ Additionally, 5-HTR2 antagonists have been shown to increase the dopaminergic neurotransmission. ${ }^{31}$ The 5-HTR2 receptors are expressed in the prefrontal cortex, the nucleus acumbens, the putamen and the caudate, as well as in the platelets of the peripheral blood. In contrast 5-HTR3 expression is restricted to many cortical and subcortical regions, such as the amygdala, the hippocampus or other areas of the limbic system. ${ }^{32}$

Williams et $a l^{6}$ studied a silent serotonin polymorphism T-102C in the 5-HTR2 receptor gene, indicating a higher than expected proportion of C-102 allele and C-102/C-102 genotype of this polymorphism in patients with schizophrenia. Furthermore, other studies have extensively focused on the possible relation of the certain HTR2 polymorphism to clozapine response in schizophrenic patients, suggesting that cases with C-102/C-102 genotype have higher N100 amplitude than other patients after clozapine treatment. ${ }^{33}$ Although this base substitution does not change the amino-acid sequence of the protein, some groups postulated that the T-102C polymorphism may act either at the translation stage through effects on stability of the $\mathrm{mRNA}^{31,34}$, or alternatively may be in linkage disequilibrium with the functional variant within the 5-HTR2 receptor gene itself, or within a contiguous locus.

In the present work we did not detect any positive correlation between schizophrenia and variant C-102, on the contrary we observed a statistically significant association between T-102 allele and the disease with OR of 2.11 $(P<0.0001)$. Estimations of the genotype frequencies also revealed considerable differences between the two groups, indicating a higher susceptibility of the T-102/T-102 genotype for schizophrenia development. Similarly, the correlation of the presence of the T-102 allele in two copies in genotype composition (wild-type homozygous) with the disease was established singularly significant, even after estimation of the ORs between wild-type homozygotes and heterozygotes using the C-102/C-102 as genotype baseline risk $(P=0.0001, \mathrm{OR}=3.59,95 \% \mathrm{CI}=1.71-7.59)$.

The comparison of the C-102 allele frequency in our control group with respective published frequencies from other European populations indicates more or less a geographical distribution. For example, Southern countries with approximate latitudes show similar C-102 frequencies ranging from 0.505 (Greece) to 0.51 (Italy, Austria). However, this frequency seems to be constantly increased, as we move to north, getting values from 0.55 (France) to 0.59 (UK) or 0.67 (Sweden). ${ }^{6}$ The frequencies of C-102 allele in patient group among different European countries follow a similar distribution pattern with minor exceptions; however, our estimation is sensibly lower than the expected. ${ }^{6}$ These observations reflect likely the differential distributions of the polymorphic gene pool between populations in various geographical sections.

Additionally, statistical analysis including clinical parameters revealed that excess heterozygosity was most pronounced in patients with an earlier onset of schizophrenic symptoms.

Summarizing, our results demonstrate a strong positive correlation of the C-102 allele of the HTR2 receptor gene polymorphism, as well as the respective mutant type genotype, with a protective genotypic advantage concerning the susceptibility or the phenotypic expression of schizophrenia disorder within the Greek population.

With regard to DRD3 allele frequencies, no significant differences were obtained between controls and patients. Nevertheless, our overall findings suggest that in our sample the genetic contribution of the A-206 allele, presented in two copies in the genotype, might play a meaningful role in the appearance of schizophrenia, as was proposed by other groups. However, it is worth to explore whether these statistical differences result from true association or from nonsignificant departure from HW distributions in controls or in cases.

Concerning the sample size, we recognize that was relative small; however, the main advantage of the present study, in contrast to the other multicentre studies, was that our population was more homogeneous, well-balanced and dispersed over a small geographical area and the results are likely to provide a more accurate estimation of the frequency of these mutations in the 5-HTR2a and DRD3 genes.

\section{References}

1 Carlsson A, Lindquist M: Effect of chloropromazine and haloperidol on formation of methoxytyramine and normetanephrine in mouse brain. Acta Pharmacol Toxol 1963; 20: $140-144$.

2 Seeman P, Lee T: The dopamine-releasing actions of neuroleptics and ethanol. J Pharmacol Exp Ther 1974; 190: 131-140.

3 Pickar D: Prospects for pharmacotherapy of schizophrenia. Lancet 1995; 345: 557-562. 
4 Williams J, Owen MJ, Spurlock G et al: Searching for susceptibility genes for schizophrenia: the European Multicentre Association Study. New Trends Exp Clin Psychiatry 1995; 11: 7-12.

5 Baron M: Genetics of schizophrenia and the new millennium: progress and pitfalls. Am J Hum Genet 2001; 68: 299-312.

6 Williams J, Spurlock G, McGuffin P et al: Association between schizophrenia and T102C polymorphism of the 5hydroxytryptamine type 2a-receptor gene. European Multicentre Association Study of Schizophrenia (EMASS) Group. Lancet 1996; 347: 1294-1296.

7 Spurlock G, Williams J, McGuffin P et al: European Multicentre Association Study of Schizophrenia: a study of the DRD2 Ser311Cys and DRD3 Ser9Gly polymorphisms. Am J Med Genet 1998; 81: 24-28.

8 Jonsson E, Lannfelt L, Sokoloff P et al: Lack of association between schizophrenia and alleles in the dopamine D3 receptor gene. Acta Psychiatr Scand 1993; 87: 345-349.

9 Yang L, Li T, Wiese C et al: No association between schizophrenia and homozygosity at the D3 dopamine receptor gene. Am J Med Genet 1993; 48: 83-86.

10 Anney RJ, Rees MI, Bryan E et al: Characterisation, mutation detection, and association analysis of alternative promoters and 5' UTRs of the human dopamine D3 receptor gene in schizophrenia. Mol Psychiatry 2002; 7: 493-502.

11 Warren Jr JT, Peacock ML, Rodriguez LC, Fink JK: An MspI polymorphism in the hyman serotonin receptor gene (HTR2): detection by DGGE and RFLP analysis. Hum Mol Genet 1993; 2: 338.

12 McGuffin P, Owen MJ, Farmer AE: Genetic basis of schizophrenia. Lancet 1995; 346: 678-682.

13 Ross CA: Schizophrenia genetics: expansion of knowledge? Mol Psychiatry 1999; 4: 4-5.

14 Sokoloff P, Giros B, Martres MP, Bouthenet ML, Schwartz JC: Molecular cloning and characterization of a novel dopamine receptor (D3) as a target for neuroleptics. Nature 1990; 347: $146-151$

15 Sokoloff P, Levesque D, Martres MP et al: The dopamine D3 receptor as a key target for antipsychotics. Clin Neuropharmacol 1992; 15: 456A-457A.

16 Guo N, Klitenick MA, Tham CS, Fibiger HC: Receptor mechanisms mediating clozapine-induced c-fos expression in the forebrain. Neuroscience 1995; 65: 747-756.

17 Jernigan TL: Neuroanatomical factors in schizophrenia; in Kavanagh DJ (ed): Schizophrenia: an overview and practical handbook. London: Chapman \& Hall; 1992.

18 Arinami T, Gao M, Hamaguchi $\mathrm{H}$, Toru $\mathrm{M}$ : A functional polymorphism in the promoter region of the dopamine D2 receptor gene is associated with schizophrenia. Hum Mol Genet 1997; 6: 577-582.

19 Himei A, Koh J, Sakai J, Inada Y, Akabame K, Yoneda H: The influence on the schizophrenic symptoms by the DRD2Ser/ Cys311 and -141C Ins/Del polymorphisms. Psychiatry Clin Neurosci 2002; 56: 97-102.
20 Crocq MA, Mant R, Asherson P et al: Association between schizophrenia and homozygosity at the dopamine D3 receptor gene. J Med Genet 1992; 29: 858-860.

21 Lannfelt L, Sokoloff P, Martres MP et al: Amino acid substitution in the dopamine D3 receptors a useful polymorphism for investigating psychiatric disorders. Psychiatr Genet 1992; 2: 249-256.

22 Griffon N, Crocq MA, Pilon C et al: Dopamine D3 receptor gene: organization, transcript variants, and polymorphism associated with schizophrenia. Am J Med Genet 1996; 67: 63-70.

23 Ventriglia M, Bocchio Chiavetto L, Bonvicini C et al: Allelic variation in the human prodynorphin gene promoter and schizophrenia. Neuropsychobiology 2002; 46: 17-21.

24 Nothen MM, Cichon S, Propping P, Fimmers R, Schwab SG, Wildenauer DB: Excess of homozygosity at the dopamine D3 receptor gene in schizophrenia not confirmed. J Med Genet 1993; 30: $708-709$.

25 Laurent C, Savoye C, Samolyk D et al: Homozygosity at the dopamine D3 receptor locus is not associated with schizophrenia. J Med Genet 1994; 31: 260.

26 Liao DL, Yeh YC, Chen HM, Chen H, Hong CJ, Tsai SJ: Association between the Ser9Gly polymorphism of the dopamine D3 receptor gene and tardive dyskinesia in Chinese schizophrenic patients. Neuropsychobiology 2001; 44: 95-98.

27 Segman R, Neeman T, Heresco-Levy U et al: Genotypic association between the dopamine D3 receptor and tardive dyskinesia in chronic schizophrenia. Mol Psychiatry 1999; 4: 247-253.

28 Mant R, Williams J, Asherson P, Parfitt E, McGuffin P, Owen MJ: Relationship between homozygosity at the dopamine D3 receptor gene and schizophrenia. Am J Med Genet 1994; 54: $21-26$.

29 Nimgaonkar VL, Zhang XR, Caldwell JG, Ganguli R, Chakravarti A: Association study of schizophrenia with dopamine D3 receptor gene polymorphisms: probable effects of family history of schizophrenia? Am J Med Genet 1993; 48: 214-217.

30 Gaitonde EJ, Morris A, Sivagnanasundaram S, McKenna PJ, Hunt DM, Mollon JD: Assessment of association of D3 dopamine receptor MscI polymorphism with schizophrenia: analysis of symptom ratings, family history, age at onset, and movement disorders. Am J Med Genet 1996; 67: 455-458.

31 Arranz M, Collier D, Sodhi M et al: Association between clozapine response and allelic variation in 5-HT2A receptor gene. Lancet 1995; 346: 281-282.

32 Nothen MM, Rietschel M, Erdmann J et al: Genetic variation of the 5-HT2A receptor and response to clozapine. Lancet 1995; 346: 908-909.

33 Yu YW, Tsai SJ, Yang KH, Lin CH, Chen MC, Hong CJ: Evidence for an association between polymorphism in the serotonin-2A receptor variant (102T/C) and increment of N100 amplitude in schizophrenics treated with clozapine. Neuropsychobiology 2001; 43: $79-82$.

34 Smith CAD, Smith G, Wolf CR: Genetic polymorphisms in zenobiotic metabolism. Eur J Cancer 1994; 30a: 1921-1935. 